\title{
Direct Synthesis of Few-Layer F-Doped Graphene Foam and Its Lithium/Potassium Storage Properties
}

Zhicheng Ju, ${ }^{* \dagger}$ Shuai Zhang, ${ }^{\dagger}$ Zheng Xing, ${ }^{, \dagger}$ Quanchao Zhuang, ${ }^{\dagger}$ Yinghuai Qiang, ${ }^{\dagger}$ Yitai Qian ${ }^{\ddagger}$

${ }^{\dagger}$ School of Materials Science and Engineering, China University of Mining and Technology, Xuzhou, Jiangsu, 221116, PR China

${ }^{\ddagger}$ Hefei National Laboratory for Physical Science at Microscale, Department of Chemistry, University of Science and Technology of China, Hefei, Anhui, 230026, PR China

*E-mail: juzc@ cumt.edu.cn

*E-mail: xzh086@cumt.edu.cn. 


\section{Sample Characterization}

The X-ray powder diffraction (XRD) measurements were carried out by a D8 Advance X-ray diffractometer of Bruker equipped with $\mathrm{Cu}$ Ka radiation $(\lambda=1.54182 \AA)$ at a scanning rate of $10^{\circ} \mathrm{min}^{-1}$. The X-ray photoemission spectroscopy (XPS) measurements were carried out by a Thermo Scientific ESCALAB 250 X-ray photoelectronic spectrometer with a non-monochromated $\mathrm{Mg} \mathrm{K} \alpha \mathrm{X}$-ray radiation as the excitation source. The Raman spectroscopy data was taken on a LABRAM-HR confocal laser MicroRaman spectrometer with excitation wavelength of $514.5 \mathrm{~nm}(2.41 \mathrm{eV})$. The specific surface areas of the sample were measured by TriStar II 3020 (Micromeritics Instrument Corporation, U.S.A.) and calculated by $\mathrm{N}_{2}$ adsorption-desorption isotherms. The morphology of the sample were measured using a JEOL JSM-6700F field emission scanning electron microscope (SEM) operating at $15 \mathrm{kV}$ and JEM 1011 transmission electron microscope (TEM) operated at $100 \mathrm{kv}$. The JEOL-2010 high-resolution transmission electron microscope (HRTEM) operated at $200 \mathrm{kV}$ were used to investigate the nanostructural characterizations.

\section{Electrochemical Measurements}

The anode performance tests were performed with a CR2025 coin-type cell, which just like we previously reported. ${ }^{1,2}$ The FFGF was used for anode electrode, and Li foil as cathode. The electrode material for coin cell was prepared by mixing accurate weighed active material (FFGF, $70 \mathrm{wt} \%$ ), Super P acetylene black as additives of conducting (15 wt $\%$ ), and polyvinylidene fluoride (PVDF, $15 \mathrm{wt} \%$ ) as binder. Above materials were dispersed in N-methyl pyrrolidone and ball milled for 5 hours with speed of $400 \mathrm{rpm}$ to obtain uniformly mixed slurry. Then the mixture was uniformly coated on a copper foil current collector and subsequently drying at $80{ }^{\circ} \mathrm{C}$ in a 
vacuum oven for 12 hours. Electrode disc with diameter of $14 \mathrm{~mm}$ was cut for electrochemical characterization. Mass loading for the electrode was determined by comparing the weight of the disc with that of the original copper foil. Typically electrode active material loading was about ca. 0.8-0.9 $\mathrm{mg}$ on one disc. The batteries were assembled in an argon-filled glovebox; Li foil was used as cathode and fluorinated graphene was applied as anode. The Celgard 2300 microporous membrane was served as polypropylene separator, and the $\operatorname{LiPF}_{6}(1 \mathrm{M})$ solution dissolved in ethylene carbonate (EC)/dimethyl carbonate (DMC) by 1:1 volume ratio as the electrolyte. The galvanostatic discharge-charge tests was tested under various rates in the voltage range of 0.01-3.0 $\mathrm{V}$ (vs. $\mathrm{Li}^{+} / \mathrm{Li}$ ) using a computer-controlled multichannel battery test unit (LAND-CT2001A battery cycler) at room temperature. The typical cyclic voltammetry (CV) measurements were carried out in the range of $0.01-3.0 \mathrm{~V}$ at a scanning rate of $0.05 \mathrm{mV} / \mathrm{s}$ by electrochemical workstation of LK-2005A (Lanlike, Tianjin China) with lithium foil employed as the counter and reference electrodes. Electrochemical impedance spectral measurements (EIS) were tested on a Potentiostat-510 connected to an FRA-520 electrochemical workstation (MaterialsMates, Italia) and executed in the range from $100 \mathrm{kHz}$ to $0.01 \mathrm{~Hz}$ with an AC signal amplitude of $5 \mathrm{mV}$. The EIS of each cycle were all carried out at Open Circuit Voltage (OCV) mode at 3.0 V. Fitting of the impedance spectra to the typical equivalent circuit were executed by the code ZSimpWin.

In PIBs, coin cells CR2025 were fit with K foil as the reference/counter electrode, a glass-fiber separator (Whatman GF/D), and 0.8 $\mathrm{M} \mathrm{KPF}_{6}$ in EC: DEC (1:1) as an electrolyte in an argon-filled glovebox. The $\mathrm{K}$ foil making procedure as following: Potassium metal foil was cut from potassium bulk (Aladdin Industrial Corporation) and then rolling flat (thickness of about $1 \mathrm{~mm}$ ) by cylindrical wooden rod. Finally, a potassium disk (the diameter of the disc is $16 \mathrm{~mm}$, and the 
thickness is $1 \mathrm{~mm}$ ) was punched from the potassium foil using a punch. The typical active mass loading was $0.8 \sim 0.9 \mathrm{mg}$. The galvanostatic charge-discharge tests were measured at various rates in the range of $0.01-1.5 \mathrm{~V}$ (vs. $\mathrm{K}^{+} / \mathrm{K}$ ). The EIS were tested on CHI660B electrochemical workstation and executed in the range from $1 \mathrm{MHz}$ to $0.01 \mathrm{~Hz}$ with an $\mathrm{AC}$ signal amplitude of 5 $\mathrm{mV}$. The EIS of each cycle were all carried out at OCV mode at $1.5 \mathrm{~V}$.

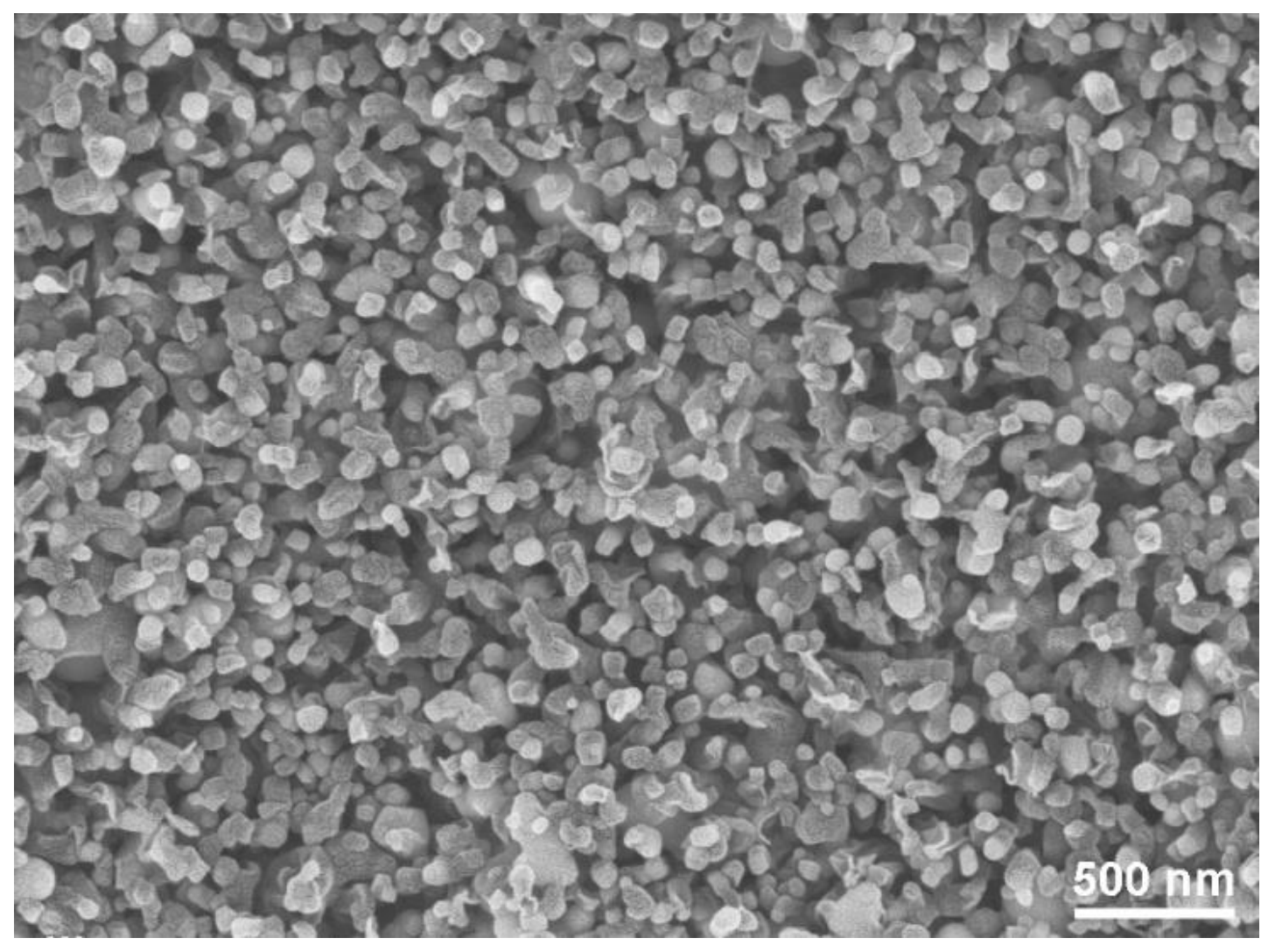

Figure S1. SEM micrograph of wrinkled graphene foam with lower magnification. 

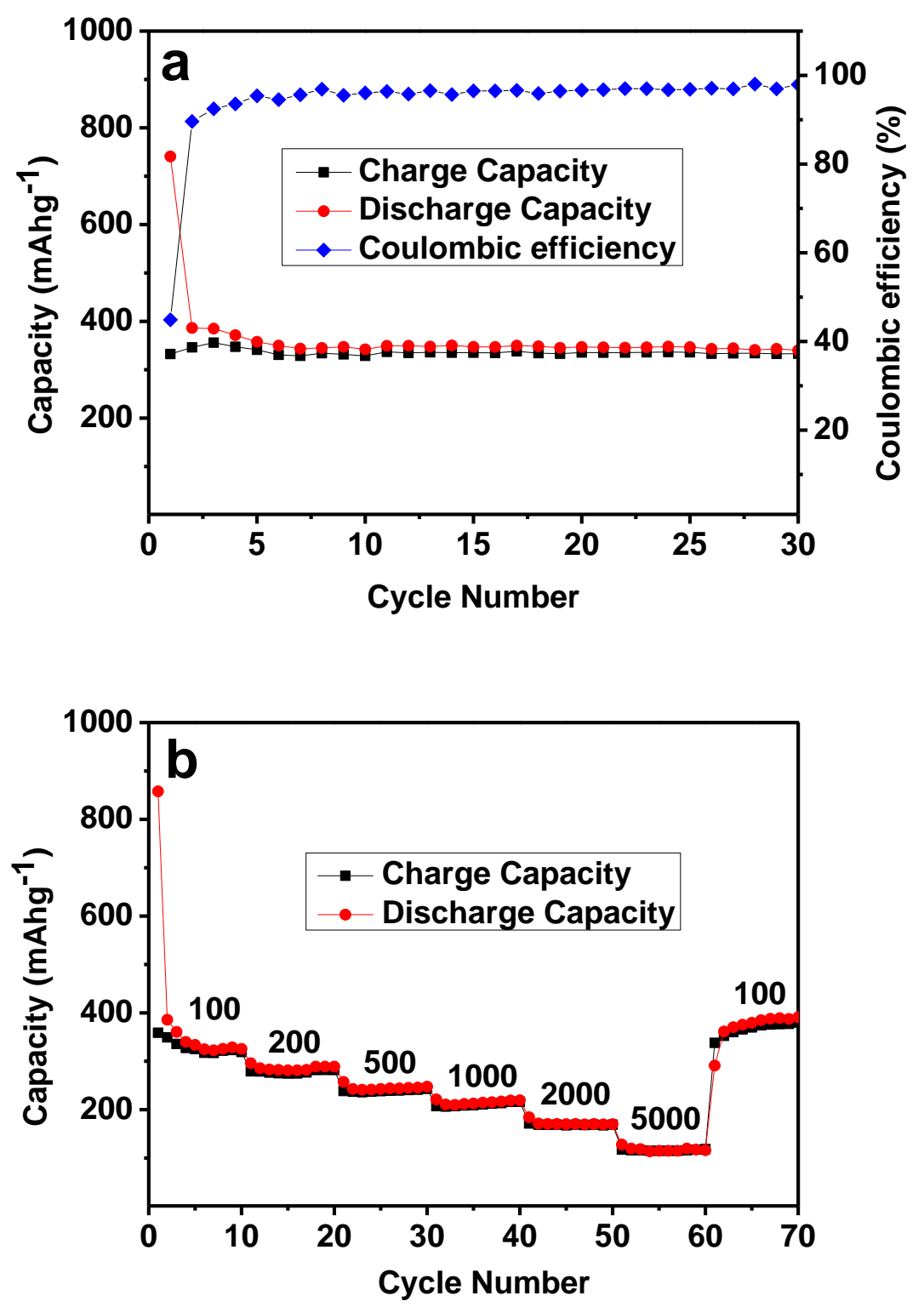

Figure S2. Electrochemical performance of the PEC/Li. (a) Discharge/charge capacity at current density of $100 \mathrm{~mA} \cdot \mathrm{g}^{-1}$. (b) Rate performance at variable current densities in the voltage range of $0.01 \mathrm{~V}$ and $3.0 \mathrm{~V}$.

Figure S2a shows the discharge-charge cycling performance of the PEC electrode at $100 \mathrm{~mA} \cdot \mathrm{g}^{-1}$. The initial discharge and charge capacities are740.8 $\mathrm{mAh} \cdot \mathrm{g}^{-1}$ and $332.5 \mathrm{mAh} \cdot \mathrm{g}^{-1}$, respectively. After the 30th cycle, the discharge capacity maintains at about $340.2 \mathrm{mAh} \cdot \mathrm{g}^{-1}$.The values is close to the theoretical capacity of graphite $\left(372 \mathrm{mAh} \cdot \mathrm{g}^{-1}\right)$. Figure S2b shows the rate capacity of the PEC electrode. 
Even in the high current of $5000 \mathrm{~mA} \cdot \mathrm{g}^{-1}$, the capacity is still more than $130 \mathrm{mAh} \cdot \mathrm{g}^{-1}$. If the current density goes back to $100 \mathrm{~mA} \cdot \mathrm{g}^{-1}$, the capacity returns to $390 \mathrm{mAh} \cdot \mathrm{g}^{-1}$, which recovers the initial capacity.
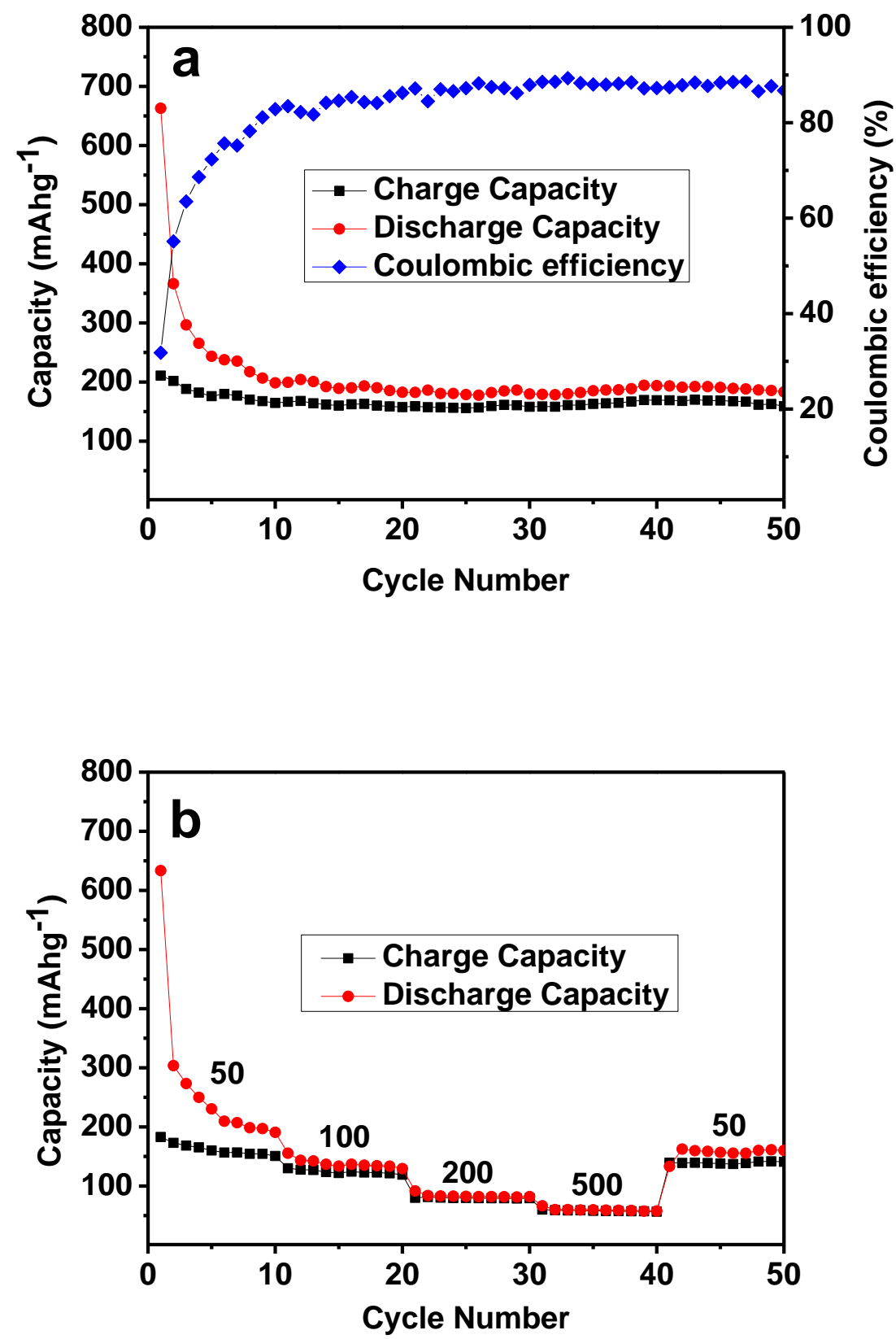

Figure S3. Electrochemical performance of the PEC/K. (a) Discharge/charge capacity at current density of $50 \mathrm{~mA} \cdot \mathrm{g}^{-1}$. (b) Rate performance at variable current densities in the voltage range of $0.01 \mathrm{~V}$ and $1.50 \mathrm{~V}$. 
Figure S3a shows the galvanostatic charge-discharge profiles of $\mathrm{PEC} / \mathrm{K}$ at a current density of $50 \mathrm{~mA} \cdot \mathrm{g}^{-1}$. The first discharge and charge capacities are 663.8 and 210.9 $\mathrm{mAh} \cdot \mathrm{g}^{-1}$, respectively. The initial coulombic efficiency of $\mathrm{PEC} / \mathrm{K}$ is $31.8 \%$, which promptly increases in the following discharge and charge process and remained about $90 \%$ after 15 cycles. After the 50th cycle, the discharge capacity maintains at about $162.8 \mathrm{mAh} \cdot \mathrm{g}^{-1}$. The rate cycling performance of $\mathrm{PEC} / \mathrm{K}$ were also investigated (Figure S3b). The electrode delivers reversible capacities of about 160.3, 125.7, 80.1 and $60.5 \mathrm{mAh} \cdot \mathrm{g}^{-1}$ at current densities of $50,100,200$, and $500 \mathrm{~mA} \cdot \mathrm{g}^{-1}$, respectively. The capacity can recover to $139.8 \mathrm{mAh} \cdot \mathrm{g}^{-1}$ when the current density decreases to 50 $\mathrm{mA} \cdot \mathrm{g}^{-1}$
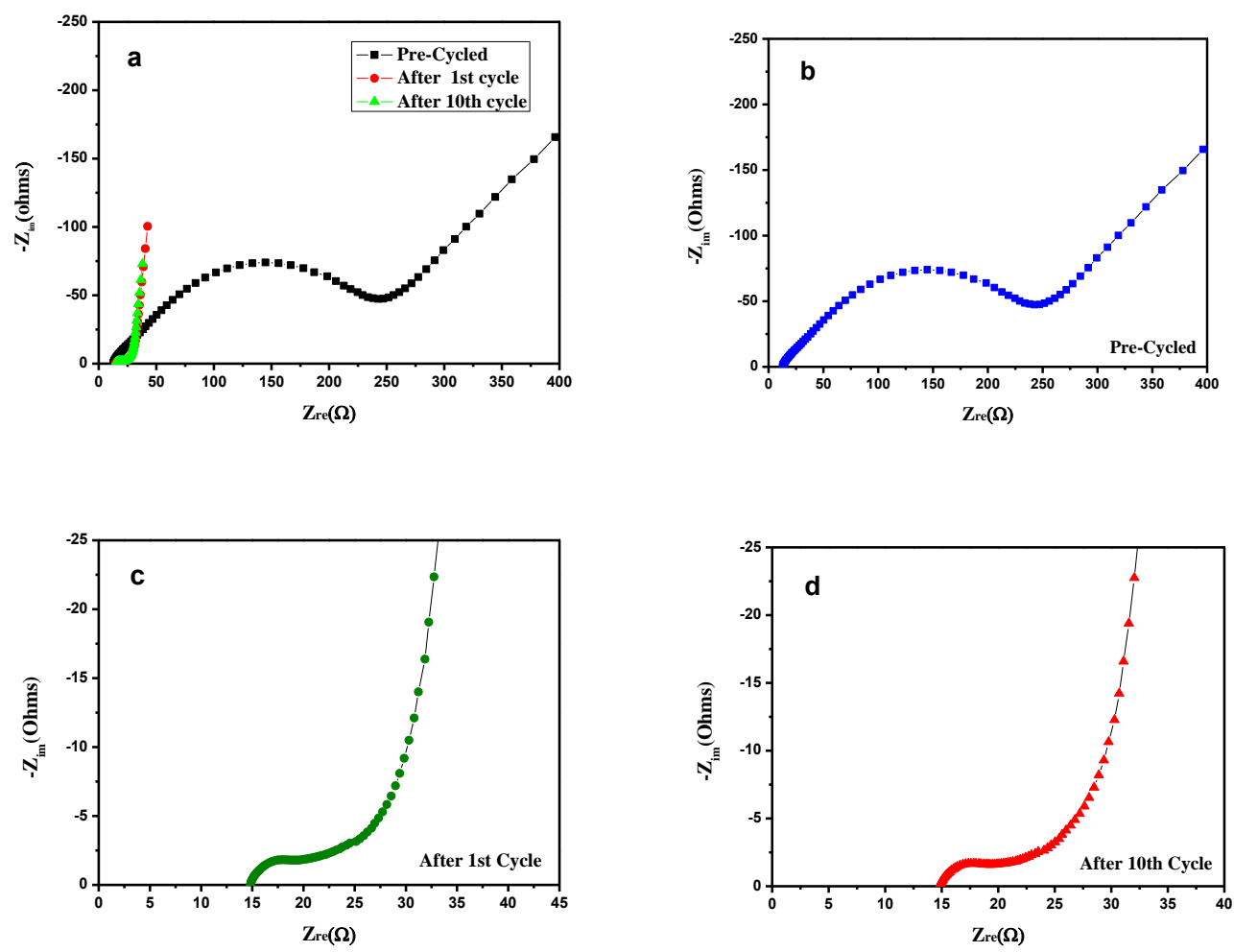

Figure S4. EIS the FFGF electrode vs $\mathrm{K} / \mathrm{K}^{+}$. (a) Nyquist plots (pre-cycled, $1^{\text {st }}, 10^{\text {th }}$ cycle), (b)

Nyquist plot of the pre-cycled electrode, (c) Nyquist plot of the electrode after $1^{\text {st }}$ cycle, (d) Nyquist plot of the electrode after $10^{\text {th }}$ cycle. 
As shown in Figure S4, the three typical Nyquist plots all consist of a semicircle in the high-frequency region and a quasi-sloping line in the low-frequency region. This means not matter for the pre-cycled or the cycled electrodes, the FFGF electrodes all possess low interfacial charge-transfer resistances. Moreover, the interfacial charge-transfer resistance of the cycled electrodes (both $1^{\text {st }}, 10^{\text {th }}$ cycle) decrease greatly comparing with the pre-cycled electrode, which indicates that the ion conductivity improvement after continuously K-ion insertion/desertion process.

\section{REFERENCES}

1. Ju, Z.; Zhang, E.; Zhao, Y.; Xing, Z.; Zhuang, Q.; Qiang, Y.; Qian, Y., One-Pot Hydrothermal Synthesis of $\mathrm{FeMoO}_{4}$ Nanocubes as an Anode Material for Lithium-Ion Batteries with Excellent Electrochemical Performance. Small 2015, 11, 4753-4761.

2. Huang, K.; Yan, C.; Wang, K.; Zhang, Y.; Ju, Z., Phases hybriding and graphene-like $\mathrm{TiO}_{2}$, for high-performance Na-ion batteries. J. Alloys. Compd. 2016, 687-683. 